\title{
Miniaturisiere photoakustische Detektoren für den Nachweis fluorhaltiger Kältemittel
}

\author{
Christian Weber $^{1,2,{ }^{*}}$, Mahmoud El-Safoury ${ }^{1, *}$, André Eberhardt ${ }^{1}$, Dr. Katrin Schmitt ${ }^{1,2}$, Prof. Dr. Jürgen \\ Wöllenstein ${ }^{1,2}$ \\ ${ }^{1}$ Fraunhofer Institut für physikalische Messtechnik IPM, 79110 Freiburg i. Br. \\ ${ }^{2}$ Institut für Mikrosystemtechnik - IMTEK, Universität Freiburg 79110 Freiburg i. Br. \\ * Beide Autoren haben im gleichen Umfang zu dieser Arbeit beigetragen. \\ christian.weber@ipm.fraunhofer.de; mahmoud.el-safoury@ipm.fraunhofer.de; \\ andre.eberhardt@ipm.fraunhofer.de; katrin.schmitt@imtek.uni-freiburg.de; \\ juergen.woellenstein@ipm.fraunhofer.de
}

\section{Kurzzusammenfassung}

Durch die Umstellung auf klimafreundliche Kältemittel im KFZ-Bereich, kam es in den letzten Jahren zu einer wachsenden Nachfrage nach Messtechnik für diese Kältemittel. Wir präsentieren im Folgenden unsere Arbeiten an photoakustischen Detektoren für die flourhaltigen Kältemittel R123a und R1234yf. Durch Simulationen konnten die Detektordimensionen optimal für beide Kältemittel ausgelegt und somit zuverlässige und empfindliche Detektorelemente geschaffen werden. Das in ersten Versuchen bestimmte Signal-zu-Rausch-Verhältnis reicht aus, um das Kältemittel R1234yf mit einer Auflösung 0,5 vol.\% über den gesamten Konzentrationsbereich zuverlässig bestimmen zu können

Keywords: Photoakustische Detektoren, Photometer, Kältemittel, R1234yf, R134a

\section{Einleitung}

Kältemittel aus mobilen und stationären Kälteanlagen sind maßgeblich an den Emissionen von fluorierten Treibhausgasen weltweit beteiligt. In KFZ Klimaanlagen betrifft dies vor allem das weit verbreitete R134a $(1,1,1,2$-Tetrafluorethan). Es weist ein Global Warming Potential (GWP) von 1430 verglichen mit $\mathrm{CO}_{2}$ auf [1] und ist aufgrund seiner Klimaschädlichkeit seit dem Jahr 2017 für Neuwagen innerhalb der EU verboten ${ }^{[2]}$. Als alternatives Kältemittel kommt seitdem das ebenfalls fluorierte R1234yf (2,3,3,3Tetrafluorpropen) zum Einsatz, welches mit einem GWP von 4 deutlich weniger klimaschädlich ist ${ }^{[1]}$. Die thermodynamischen Eigenschaften beider Kältemittel ähneln sich sehr stark, weshalb die gleichen KältekreislaufKomponenten zum Einsatz kommen können. Da jedoch die Öle und Dichtungen der Kälteanlagen auf das jeweilige Kältemittel hin optimiert sind, gilt es im Servicefall das Kältemittel eindeutig zu identifizieren, um eine Falschbefüllung zu verhindern. Daneben sollen auch Verunreinigungen der Kältemittel erkannt werden. Das gilt besonders, wenn ein Teil des wesentlich günstigeren R134a dem R1234yf zugemischt wird um mit dieser Mischung den Kältekreislauf zu befüllen.

In diesem Artikel beschreiben wir den Aufbau und die Auslegung photoakustischer NDIR-
Detektoren zum optischen Nachweis und der eindeutigen Trennung beider Kältemittel. Die Detektoren sind dazu geeignet in einem ZweiKammer-System eingesetzt zu werden, welches nur die Zielgase zur optischen Filterung verwendet und somit keine weiteren Filter benötigt. Somit dienen die Detektoren als kostengünstige Grundlage für ein sensitives Messsystem zur Konzentrationsbestimmung dieser Kältemittel.

\section{Grundlagen und Messprinzip}

Die entwickelten Detektoren beruhen auf dem potoakustischen Effekt zur Intensitätsmessung von Infrarotstrahlung in gasspezifischen Wellenlängenbereichen. Der Effekt wurde 1880 von Alexander Greyham Bell erstmalig publiziert ${ }^{[3]}$. Er beschreibt den Zusammenhang zwischen der Absorption von Strahlung in abgeschlossenen Gasvolumina und des darauffolgenden Druckanstiegs, der als Schallwelle wahrgenommen werden kann. Stimmt die Photonenenergie der Infrarotstrahlung mit der Energie eines möglichen Schwingungs-RotationsÜberganges der Gasmoleküle überein, so kann das Photon absorbiert und dabei der Zustandsübergang angeregt werden ${ }^{[4]}$. Durch nichtstrahlende Relaxation des Zustandes wird die absorbierte Energie in Wärme umgewandelt, die im angeschlossenen 
Gasvolumen zu einem Druckanstieg führt, der sich im dynamischen Fall als Schallwelle im Gas zeigt. Über ein Mikrofon lässt sich die Amplitude der Schallwelle sehr sensitiv nachweisen, weshalb sich der Effekt messtechnisch nutzen lässt.

Der photakustische Effekt eignet sich direkt zur Bestimmung von Gaskonzentrationen, da die absorbierte Leistung über das LambertBeer'sche Gesetz direkt proportional zur Konzentration des Zielgases ist.

Da die Energien der Schwingungs-RotationsÜbergänge für jedes Molekül spezifisch sind, lässt sich über die Auswahl des infraroten Spektrums, eine Selektivität des Sensors auf das Zielgas erreichen. Für Breitbandquellen, wie thermische Emitter, wird dies in der Regel über optische Filter realisiert.

Der hier beschriebene Detektor arbeitet nach dem 2-Kammer-Prinzip, das heißt, er detektiert die Gaskonzentration indirekt als Detektor eines Absorptionsspektrometers (vergl. Abbildung 1). Dazu wird das Zielgas hermetisch im Detektor eingeschlossen, womit er über die fixierte Konzentration eine definierte Sensitivität auf Infrarotstrahlung aufweist. Die Besonderheit gegenüber anderen Infrarotdetektoren ist dabei die mit dem Absorptionsspektrum des eingeschlossenen Gases verknüpfte, spektral spezifische Detektorantwort.

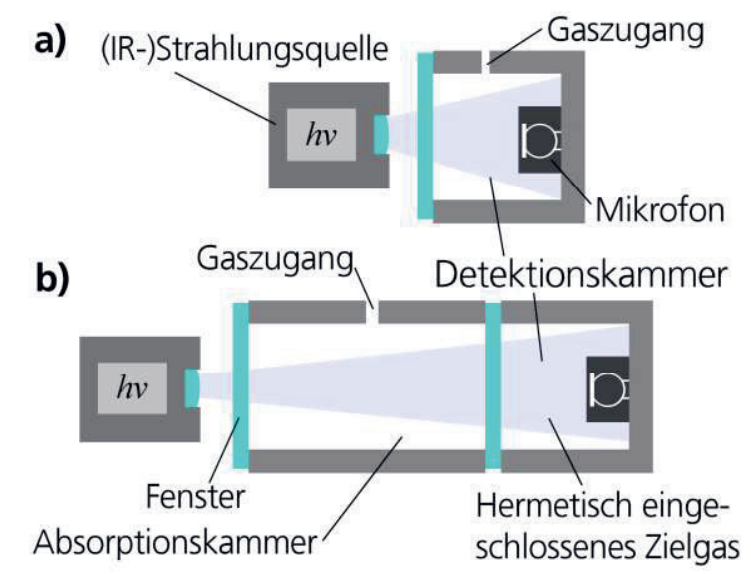

Abb. 1: Vergleich des Ein-Kammer-Prinzips (a) mit dem Zwei-Kammer-Prinzip (b) bei photoakustischen Messsystemen. Bei (a) tritt das zu messende Gas direkt in die Detektionskammer ein, während bei (b) das Zielgas in dieser Kammer hermetisch eingeschlossen ist. Das Gas wird somit als Strahlungsdetektor verwendet, mit dem die Reststrahlung, selektiv nach Durchtritt durch die Absorptionskammer, gemessen wird.
Der Detektor ist über den photoakustischen Effekt nur für die Spektralanteile empfindlich, in denen das Zielgas absorbiert.

\section{Absorptionsspektren der zu messenden Kältemittel}

Die Auslegung der photoakustischen Detektoren wurde mittels numerischer Verfahren simuliert. Die Grundlage dieser Simulationen bilden hochauflösende Infrarotspektren beider Kältemittel mit quantitativer Aussagekraft. Da diese in der Literatur nur mit unzureichender Genauigkeit zu finden waren, wurden sie mittels FTIRSpektroskopie bestimmt. Dazu wurden Konzentrationen von 0,5 bis 100 vol. $\%$ der Kältemittel in einer $10 \mathrm{~cm}$ Küvette eingestellt und die Absorptionsspektren vermessen. Anschließend wurden daraus die spektralen Absorptionskoeffizienten von R1234yf und R134a errechnet. In Abbildung 2 sind die spektralen Absorptionskoeffizienten beider Kältemittel, über der IR-Wellenlänge auftragen, dargestellt.

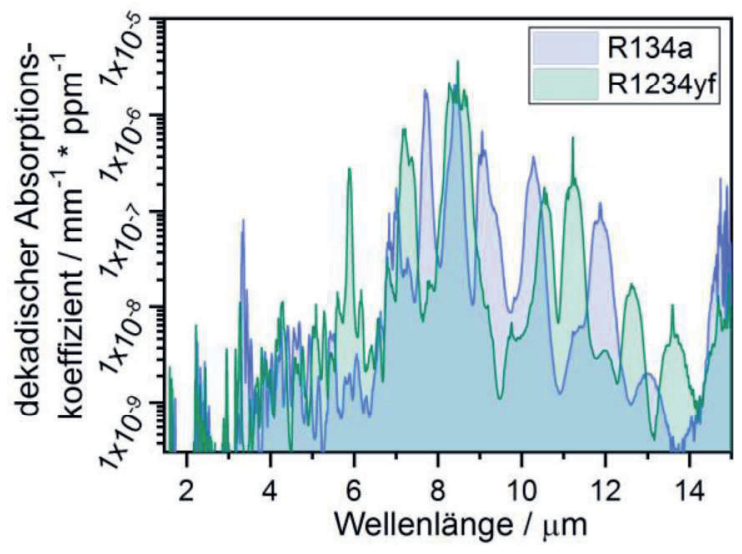

Abb. 1: Spektrale Absorptionskoeffizienten von R134a und R1234yf über der Wellenlänge der anregenden Infrarotstrahlung aufgetragen.

Die Bestimmung der Koeffizienten zeigt, dass beide Kältemittel im gesamten Bereich von 3 bis $15 \mu \mathrm{m}$ Infrarotstrahlung absorbieren. Sowohl bei R1234yf als auch bei R134a liegt die Hauptabsorptionsbande bei ca. 8,5 $\mu \mathrm{m}$. Daneben zeigen sich mehrere starke Banden zwischen 7 und $12 \mu \mathrm{m}$. Die Auffächerung in einzelne Absorptionslinien ist, besonders im Langwelligen, sehr gering ausgeprägt. Mit Ausnahme des Q-Zweigs, zeigen sich die starken Banden solide und detailarm.

\section{Simulationen zur Auslegung der Detektoren}

Die optimalen der Detektordimensionen konnte für beide Kältemittel über numerische Simulationen der Infrarotabsorption bestimmt 
werden. Um die Komplexität des photoakustischen Effektes auszuklammern, wurde sich auf die spektrale Leistungsdichte, welche im Detektor absorbiert wird, beschränkt. Quantitative Aussagen zur absoluten Druckamplitude im Detektor selbst lassen sich so nur eingeschränkt über Vergleiche des Simulationsmodells mit Messungen an bereits vorhandenen, mit anderen Gasen gefüllten, Detektoren machen. Hauptziel der Simulationen war es, eine möglichst hohe Sensitivität auf das jeweilige Ziel-Kältemittel zu erreichen, während nur geringe Querempfindlichkeiten auf das andere Kältemittel und andere Umgebungsgase vorhanden sind. Dabei muss der Detektor das Zielgas über den gesamten Bereich von 0 bis $100 \%$ detektieren können.

Da die optimale Detektorgeometrie auch vom Rest der Messtrecke abhängt, wird er innerhalb eines sogenannten Drei-KammerSystems simuliert. Dabei wird das ZweiKammer-Prinzip um eine weitere Kammer erweitert, die sich zwischen Strahlungsquelle und Absorptionskammer (vergl. Abb. 1) befindet, ergänzt.

Diese Filterkammer kann Gase (sog. Filtergase) enthalten, gegenüber denen Querempfindlichkeiten verringert werden sollen.

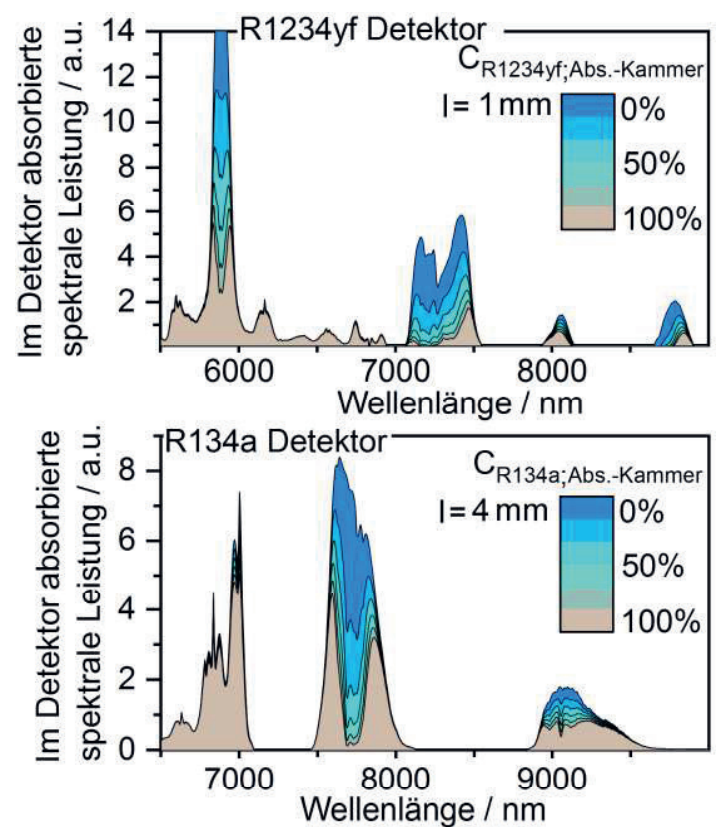

Abb. 3: Berechnete spektrale absorbierte Leistung im Detektor mit R1234yf und R134a unter verschiedenen Konzentrationen des Zielgases in der Absorptionskammer (Länge: $1 \mathrm{~mm}$ bzw. $4 \mathrm{~mm}$ ). In beiden Fällen war der Detektor rund $3 \mathrm{~mm}$ lang und hatte einen Durchmesser von $8 \mathrm{~mm}$.
Dies funktioniert, in dem in der Filterkammer alle Spektralbereiche entfernt werden, die für Querempfindlichkeiten auf die Filtergase verantwortlich sind. Folglich werden die Bereiche, in denen kein spektraler Überlapp zwischen Filter- und Zielgas vorhanden ist, am stärksten gewichtet.

Als Strahlungsquelle wurde in den Simulationen ein thermischer Strahler mit 2x2 mm strahlender Fläche, einem Emissionsgrad von 0,9 und einer Temperatur von $700^{\circ} \mathrm{C}$ angenommen.

Die Simulationen zeigen, dass bereits $3 \mathrm{~mm}$ Absorptionslänge in der Detektorkammer ausreichen, um die einfallende Strahlung im Bereich der Hauptbanden vollständig zu absorbieren. Als Durchmesser für die Absorptionskammer wurden $8 \mathrm{~mm}$ gewählt, um das Mikrofon später gut integrieren zu können. Die optimale Absorptionskammerlänge liegt im Fall von R1234yf bei $1 \mathrm{~mm}$ und bei R134a bei 4 $\mathrm{mm}$. Zur Verringerung der Querempfindlichkeit auf das jeweils andere Kältemittel wurden je $50 \mathrm{~mm}$ lange Filterzellen verwendet. Sie wurde im Fall des R1234yf Detektors mit 100\% R134a simuliert und im Fall des R134a Detektors mit 10\% R1234yf.
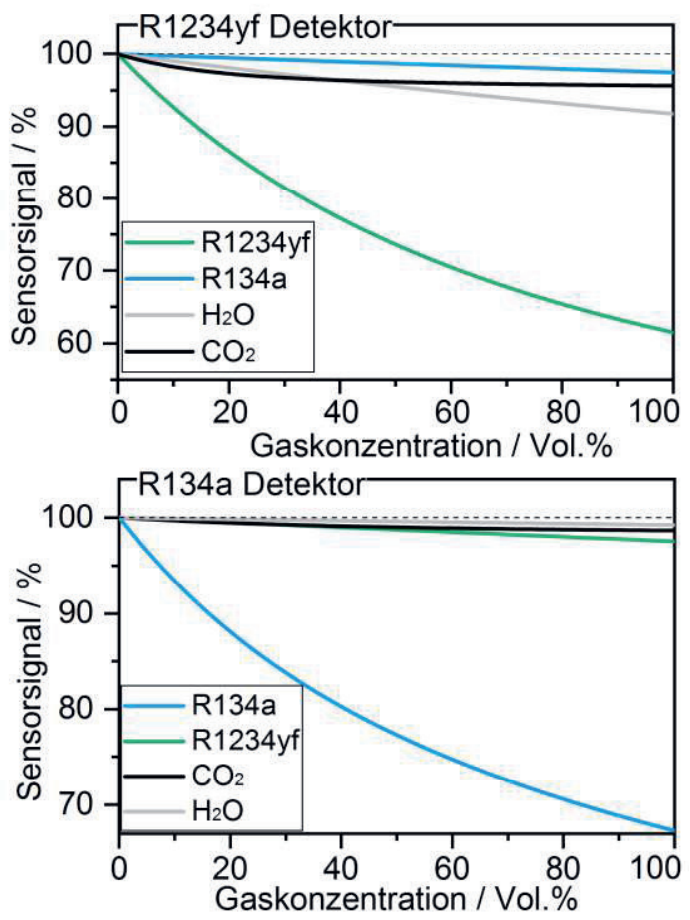

Abb. 4: Simulierte Gasreaktion der Detektoren auf die beide Kältemittel, Lufteuchte und $\mathrm{CO}_{2}$. Die Dimensionen und die Filterzellenfüllung sind analog $\mathrm{zu}$ denen in Abb. 3. 
In Abbildung 3 sind die simulierten spektralen, im Detektor absorbierten Leistungen bei Variation der Gaskonzentration in der Absorptionskammer dargestellt. Durch die Filterzelle trägt die Hauptbande bei $8,5 \mu \mathrm{m}$ in beiden Fällen kaum zum Detektorsignal bei. Beim R1234yf Detektor wird die Änderung des Gassignals hauptsächlich durch die Banden bei 5,8 und $7,3 \mu \mathrm{m}$ bestimmt. Zwischen 5 und $7 \mu \mathrm{m}$ liegt ein Spektralbereich, der zwar zum Gesamtsignal beiträgt, für die Gasreaktion aber unerheblich ist.

Die Änderung des Detektorsignals des R134a Detektors wird fast ausschließlich durch die Bande bei $7,7 \mu \mathrm{m}$ bestimmt. Auch hier tragen Banden $<7 \mu \mathrm{m}$ stark zum Gesamtsignal bei, reagieren aber kaum auf das Gas in der Absorptionskammer.

Um die Empfindlichkeiten und QuerEmpfindlichkeiten zu vergleichen, wurde in weiteren Simulationen die Reaktion der Detektoren auf beide Kältemittel und die Umgebungsgase $\mathrm{H}_{2} \mathrm{O}$ und $\mathrm{CO}_{2}$ betrachtet. Ausgewertet wurde die integrale, im Detektor absorbierte Leistung. Die Simulationsergebnisse sind in Abb. 4 gegen die jeweiligen Gaskonzentrationen aufgetragen dargestellt.

Die simulierte Empfindlichkeit des R1234yf Detektors auf R1234a ist mit $0,35 \%$ pro vol. \% ca. 17-mal stärker als die auf R134a. Bei hohen Konzentrationen sinkt sie bis auf 0,07\% pro vol.\% ab, bleibt dabei aber noch über der Empfindlichkeit auf R134a, die konstant bei 0,012\% pro vol.\% liegt. Die Empfindlichkeit des Detektors auf $\mathrm{CO}_{2}$ ist mit bis zu 0,1\% pro vol\% bei niedrigen Konzentrationen verhältnismäßig hoch. Im Falle einer Kalibrierung mit Umgebungsluft sollte der Filterzelle daher $\mathrm{CO}_{2}$ zugegeben werden.

Insgesamt zeigen die Simulationen, dass es mit beiden Detektoren in diesen Dimensionen möglich ist, die Kältemittel zuverlässig zu trennen und auch quantitativ zu messen.

\section{Aufbau des Detektors}

Ausgehend von den Maßen die in den Simulationen bestimmt wurden, konnten die Detektoren auf Basis eines TO-5 Gehäuses hergestellt werden. Die elektrische Kontaktierung wurde über sechs Drähte realisiert, die durch Glasdurchführungen im Sockel eingelassen sind (Sonderanfertigung der Fa. SIMEK; IImenau).

Zusätzlich befinden sich im Sockel noch zwei Rohre, über die eine Befüllung des Detektors mit den Kältemitteln möglich wird. Im Detektor dient eine Platine mit 0,8 mm Stärke als

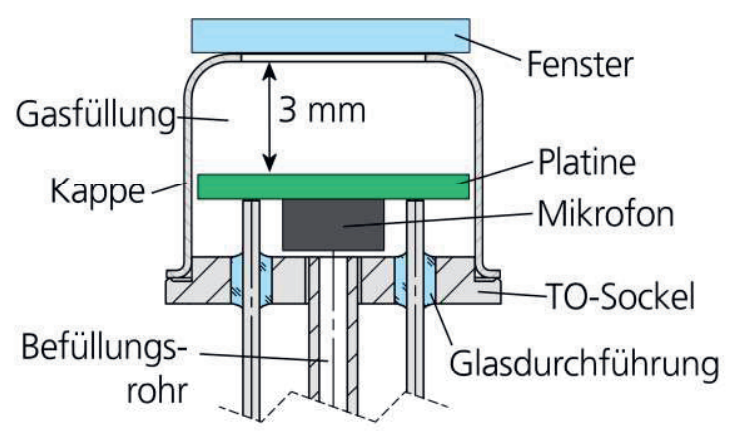

Abb. 5: Querschnitt durch den Detektoraufbau basierend auf einem TO-5 Gehäuse. Das Mikrofon wird über eine Platine auf die Drähte aufgelötet. Es handelt sich um ein Bottom-Port Mikrofon (ICS-40720 der Firma TDK)

elektrische Verbindung zwischen einem SMD MEMS-Mikrofon (ICS-40720 von TDK) und den Drähten der Glasdurchführung. Der Schallzugang zum Mikrofon findet seitlich über eine Fräsnut in der Platine statt (Siehe Abbildung 6).

Die gesamte photoakustische Detektorkammer wurde über das Auflöten einer TO-Kappe mit einem ebenfalls aufgelöteten Fenster aus unterseitig metallisiertem Calciumfluorid realisiert.
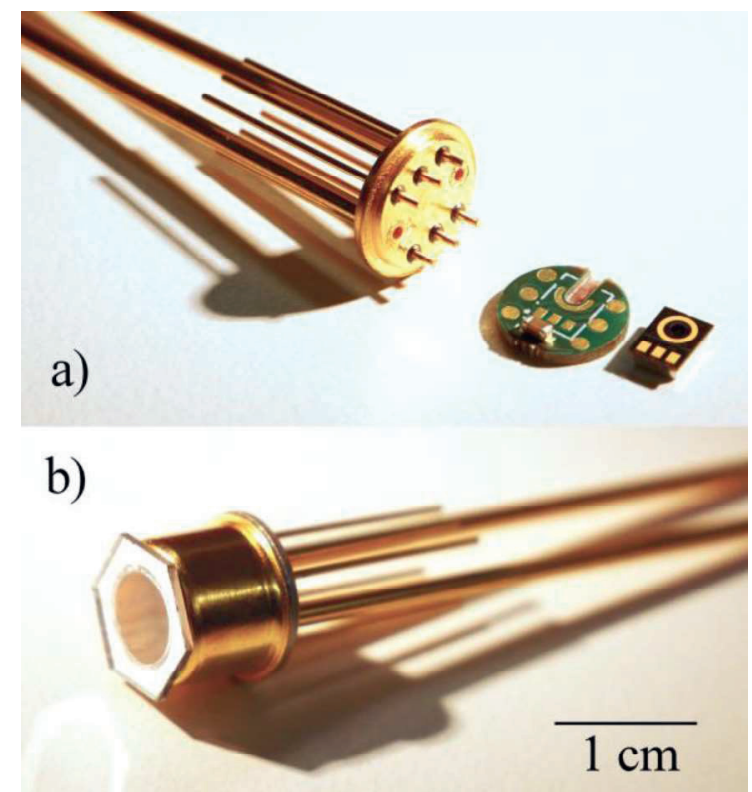

Abb. 6: Fotos des TO-Sockels mit Platine und Mikrofon vor dem Zusammenbau (a) und des aufgebauten Detektors, inklusive aufgelötetem $\mathrm{CaF}_{2}$-Fenster (b). Der Schallzugang zum Mikrofon findet über die Fräsnut in der Platine statt. 
In Abbildung 5 ist der Querschnitt durch den gesamten Detektoraufbau dargestellt.

Alle Lötverbindungen von Fenster und Elektronik konnten in einem Reflow-Löt-Schritt hergestellt werden. In Abbildung 6 ist ein Foto des fertig verlöteten Detektorgehäuses zu sehen.

\section{Befüllung des Detektors mit den Kältemitteln}

Vor der Füllung des Detektors mit den Kältemitteln wurde er bei $110^{\circ} \mathrm{C}$ für $24 \mathrm{~h}$ mit reichen Stickstoff gespült. Dies dient in erster Linie dazu, ad- und absorbierte Atmosphärengase, insbesondere Wasser, aus dem Detektor zu entfernen. Ein zusätzlicher Anteil infrarotaktiver Fremdgase würde die Querempfindlichkeiten auf diese signifikant erhöhen.

Um den photoakustischen Effekt während der Gasfüllung abschätzen zu können, wurde der Detektor in einen Versuchsaufbau eingebaut (Abbildung 7). In diesem steht er einem thermischen Strahler gegenüber (JSIR-350-4 Fa. MicroHybrid). Der Strahler wird mit $250 \mathrm{~Hz}$ von einer eigens angefertigten Elektronik sinusförmig moduliert. Gleichzeitig wird das Mikrofonsignal des Detektors über die Elektronik aufgenommen und nach einer LockIn-Filterung ausgegeben. In Abbildung 8 ist das gefilterte Detektorsignal während der Befüllung mit R1234yf gegen die Dauer der Befüllung aufgetragen.

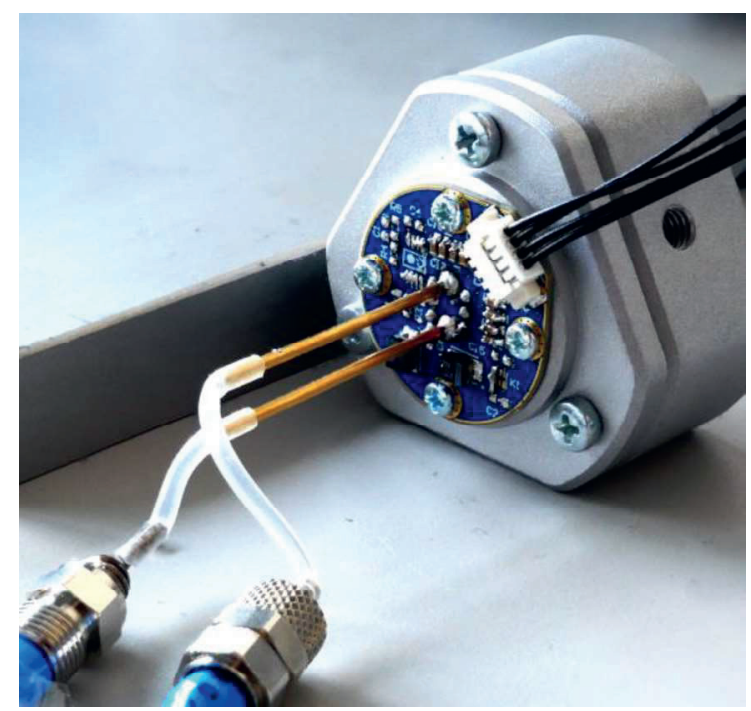

Abb. 7: Versuchsaufbau während der Befüllung des Mikrofons mit R1234yf. Der Detektor ist innerhalb des Aluminium-Gehäuses. Dem Detektor direkt gegenüber ist ein thermischer Strahler angeordnet.
Zum Zeitpunkt $t=0$ wird der Gasfluss im Detektor von reinem Stickstoff auf R1234yf umgeschaltet. Etwa $100 \mathrm{~s}$ nach dem Umschalten erreicht das Kältemittel den Detektor. Das Signal steigt durch den photoakustischen Effekt während der Befüllung stetig an und erreicht ab etwa $1200 \mathrm{~s}$ einen stationären Wert, ab dem davon ausgegangen werden kann, dass der Detektor komplett mit $\mathrm{R} 1234 y$ yefüllt ist.

Vor der Füllung mit R1234yf zeigt der Detektor bereits ein Signal, das in etwa $8 \%$ des Signals mit Kältemittel entspricht. Dieses Nullsignal kann durch die Strahlungsabsorption an der Detektorstruktur und der damit einhergehenden Photoakustik erklärt werden.

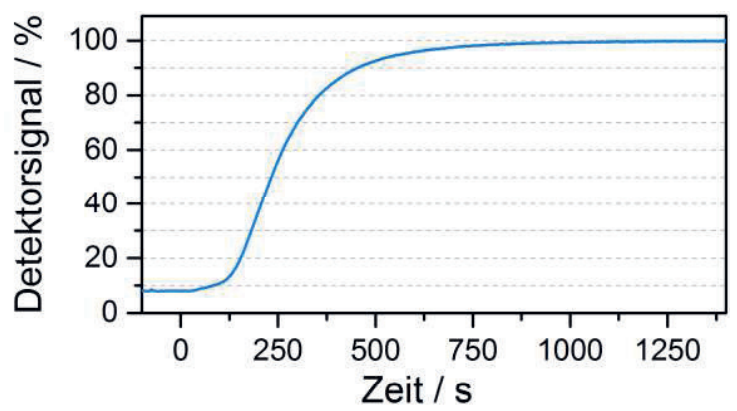

Abb. 8: Detektorsignal bei der Befüllung gegen die Zeit aufgetragen. Zum Zeitpunkt $t=0$ wird der Gasfluss von Stickstoff auf R1234yf umgeschaltet.

Nach der Befüllung des Detektors mit R1234yf, wurden die Befüllungsrohre mithilfe eines Schraubstockes abgequetscht und anschließend mit Lötzinn verschlossen, um die hermetische Dichtigkeit der Gasfüllung zu gewährleisten.

\section{Bestimmung von Stabilität und Rauschen des Detektorsignals}

Als Maß für die Signalqualität wurde das Detektorsignal über $64 \mathrm{~h}$ mit Umgebungsluft zwischen thermischen Emitter und R1234yfDetektor aufgezeichnet. Der Emitter wurde mit $250 \mathrm{~Hz}$ sinusförmig moduliert und der Abstand zum Detektor betrug bei dieser Messung $5 \mathrm{~cm}$. Sie fand unter Laborbedingungen statt, wobei die Umgebungstemperatur eine maximale Variation von $4,9^{\circ} \mathrm{C}$ (Mittelwert $20,9^{\circ} \mathrm{C}$ ) aufwies. Die Mittelungszeit des Lock-In-Filters wurde auf $1 \mathrm{~s}$ eingestellt und die Daten alle $0,5 \mathrm{~s}$ ausgelesen.

Abbildung 9 zeigt die aufgenommenen Daten der Messung im Zeitbereich und die daraus errechnete Allan-Abweichung. In den ersten 30 Minuten erwärmt sich die Messkonfiguration 
durch das Betreiben des thermischen Emitters um $11,4^{\circ} \mathrm{C}$ auf knapp $31^{\circ} \mathrm{C}$. Dabei sinkt das Detektorsignal um ca. 1,5\%, verglichen mit dem Anfangswert, ab. Im weiteren Verlauf, driftet das Temperatursignal um ca. $0,7 \%$, was größtenteils auf die Schwankungen der Umgebungstemperatur zurückzuführen ist. Ein durchgängiges Abnehmen des Signals, was auf eine Undichtigkeit des Detektors hindeuten würde, ist nicht zu beobachteten. Somit kann ein signifikantes Gasleck der Detektorkammer ausgeschlossen werden.

Die aus den Daten extrahierte Allan Abweichung ${ }^{[5]}$ zeigt, dass bei der Datenrate von $0,5 \mathrm{~s}$ die dreifache Standardabweichung und
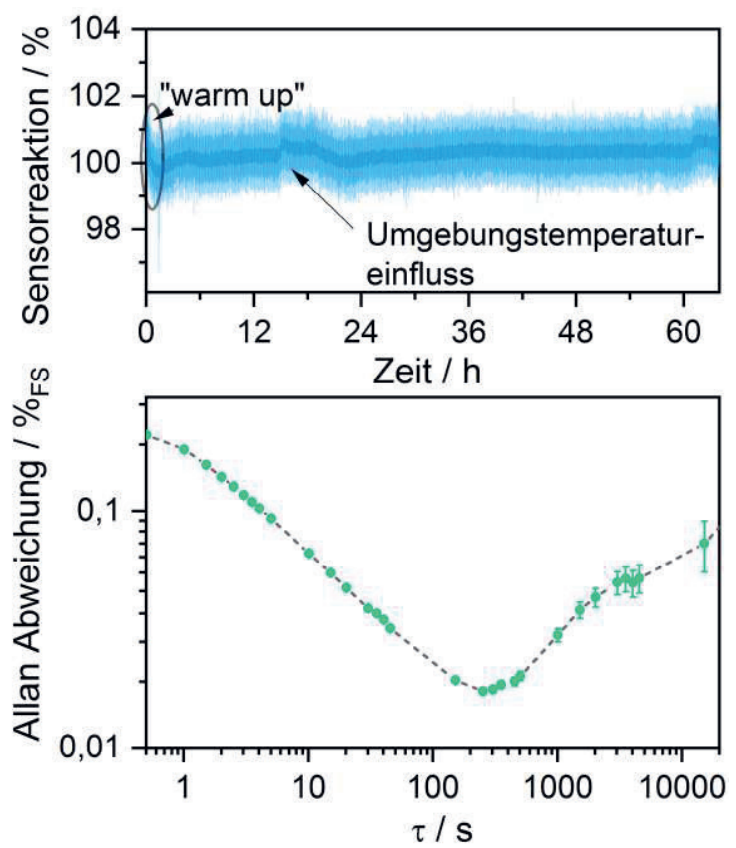

Abb. 9: Ergebnisse der Signalqualitätsmessung am R1234yf Detektor. Oben: Über $64 \mathrm{~h}$ aufgenommenes Detektorsignal mit Umgebungs/uft zwischen thermischem Emitter und Detektor. Unten: Daraus abgeleitete dreifache Allan Abweichung.

damit die Auflösungsgrenze bei ca.0,66 \% des "Fullscale"-Signals liegt. Unter der Annahme der simulierten Empfindlichkeit, reicht eine Mittelungszeit von acht bis zehn Sekunden um die geforderte Sensorauflösung von 0,5 vol.\% R1234yf zuverlässig zu erreichen.

Die auf die Temperatur zurückzuführende Detektordrift muss im späteren Messsystem über einen Temperatursensor kompensiert werden, oder sie kann über eine Temperaturstabilisierung der Messeinheit vermieden werden.

\section{Zusammenfassung}

Ziel dieser Arbeit war die Auslegung photoakustischer Detektoren für die Kältemittel R134a und R1234yf. Anhand von Simulationen wurden die wichtigsten Detektorabmessungen festgelegt und die Sensorperformance abgeschätzt. Durch ein speziell angefertigtes TO-Gehäuse konnte ein Detektor realisiert werden, der mit dem Kältemittel R1234yfgefüllt wurde. Erste Messungen zeigen ein vielversprechendes Signal-zu-RauschVerhältnis für die Reinheitsbestimmung der Kältemittel.

\section{Ausblick}

In Zukunft soll neben dem Detektor für R1234yf auch ein Detektor für R134a befüllt und vermessen werden. Anschließend erfolgt die Integration beider Detektoren in ein Messsystem, das in der Lage sein soll die Reinheit beider Kältemittel zuverlässig zu bestimmen.

\section{Danksagung}

Die durchgeführten Arbeiten wurden vom Bundesministerium für Bildung und Forschung im Rahmen des KMU-Innovativ Verbundprojektes CONTECT-R finanziert.

\section{Literatur}

[1] B. Minor, M. Spatz, HFO-1234yf low GWP Refrigerant Update. In: InternationalRefrigeration and Air Conditioning Conference at Purdue, West Lafayette, IN,USA, 2008, Paper No. 2349.

[2] Richtlinie 2006/40/EG des europäischen Parlaments und des Rates, 17.06.2006 über Emissionen aus Klimaanlagen in Kraftfahrzeugen und zur Änderung der Richtlinie 70/156/EWG des Rates

[3] A. G. Bell, "On the Production and Reproduction of Sound by Light," Am. J. Sci., vol. XX, no. 118, pp. 305-324, 1880.

[4] Z. Bozóki, A. Pogány, G. Szabó: "Photoacoustic In-struments for Practical Applications: Present, Poten-tials, and Future Challenges", Applied Spectroscopy vol. 46; 01/2011

[5] P. Werle, R. Mücke, and F. Slemr, "The limits of signal averagingin atmospheric trace gas monitoring by tunable diodelaser absorption spectroscopy," Appl. Phys. B 57, 131-139 (1993). 